\title{
Enhancing black belts via academic mastery
}

\author{
Dr Barbara M Savage \\ Senior Lecturer, HP Client Manager \& Course Leader \\ Department of Operations and Systems Management \\ Portsmouth Business School, University of Portsmouth, \\ Richmond Building, Portland Street, Portsmouth PO1 3DE, UK \\ Barbara.Savage@port.ac.uk \\ Mr Sampath K Sreevathsan \\ Lean Six Sigma Lead for EMEA, Enterprise Group Business \\ Hewlett Packard Enterprise
}

\begin{abstract}
Purpose: In May 2010, a new collaborative initiative was launched between the Portsmouth Business School (PBS) and Hewlett Packard (HP), wherein trained Black Belts were enabled to pursue an MSc in Strategic Quality Management. Five years on from its commencement, this paper explores the development and impact of this initiative.

Methodology: Inductive, exploratory, multi-viewpoint participant observer case study that triangulates the academic, student and employer reactions to the programme.

Findings: The paper evaluates how the academic dimension provided by the MSc has impacted on candidates' work as Black Belts.

Practical Implications: Employee engagement and enthusiasm has been increased, as has the depth and breadth of the knowledge base among the participating Black Belts, who are then applying these new skills in their improvement projects to make them more sustainable as well as financially valuable. They have been able to transfer knowledge to team members.

Originality/Value: This approach provides a model for accelerated development of groups of quality professionals within larger organisations. There is evidence that the community spirit that grows within and across cohorts has a multiplying effect that enhances the impact for the sponsoring organisation beyond the simple sum of the performance and skills improvement on a student by student basis.
\end{abstract}

Keywords: Lean Six Sigma, Academic Development, Sustainable Development Article Classification: Case study

\section{$1 \quad$ Introduction}

This paper explores the development and impact of a collaborative scheme between the Hewlett Packard company (HP) and the University of Portsmouth Business School (PBS), wherein an existing MSc degree course was adapted to supplement the HP Black Belt training programme and thus provide a developmental pathway beyond that training. The material presented is an experiential case study, based on the observations of the authors, who have been involved in developing the scheme since its inception in 2009 and launch in May 2010.

HP has a Total Customer Experience and Quality (TCE\&Q) Strategy which is based around a simple three-step set of aims: Execute fundamentals; Make it easy; Transform customer relationships. As a means of delivering these aims, HP has developed a company- 
wide Lean Six Sigma (LSS) programme which has been in place for more than 15 years. However, they still sought to increase the impact of this programme within the organisation and wanted to shift LSS to the strategic level. A key plank in achieving these goals was the development of methods of enhancing the capability of key talent within the company, with a particular focus on the pool of LSS Black Belts (BB).

\section{Methodology}

The methodology used for this evaluation is a longitudinal, multi-viewpoint, participant-observer study. This is based on the strategy of Grounded Theory, approached via an inductive, exploratory study of the phenomenon of the HP version of the MSc SQM (Bryman \& Bell, 2011; Saunders et al., 2012). As a consequence of this research strategy, this paper does not follow the common pattern of commencing with a review of literature. In inductive research, such as this, it is important to ensure that the interpretation of observations should not be biased by setting expectations and assumptions arising from other studies, as reported in the literature. These risks are twofold: first, that there might be a tendency to attempt to "force fit" observations to be consistent with findings reported previously; and second, that new or deviant/variant observations could be missed because they did not fit the pattern expected. Thus, literature is only brought into the paper at the discussion stage, when points actually exhibited by the phenomenon (the HP MSc SQM) are evaluated.

The programme under review has been operational for six years. Two of the reviewers have been involved throughout that period and also played key roles in the pre-launch development.

The three viewpoints are:

a. Academic. This is primarily provided by the Course Leader, who fulfils two roles in the scheme; firstly, its management and operation, and secondly, as part of the team of university staff engaged in delivery of teaching, tutoring and assessment. This individual viewpoint is supplemented by input from other members of the course team and facts from academic records.

b. Corporate Sponsor/Customer. The representative of the company responsible for creating, justifying, promoting and funding the programme within HP. This singular viewpoint is supplemented by input from company-wide sources, such as managers of students and corporate records.

c. Students. The population of past and present HP students on the MSc. The primary method was the use of an adapted version of the standard Unit and Course Feedback questionnaires used by the University of Portsmouth to monitor all courses across the University's provision. This allowed a degree of comparison with other versions of the MSc. These questionnaires were administered to all HP students at four points during the programme. Independently, students were offered less formal opportunities within HP's Black Belt community to discuss and evaluate their experiences and the deliverables and new potential arising from their involvement in the course. Additional evidence is drawn from personal conversations with some of the students involved and from HP's internal metrics.

These sources provide a rich selection of largely qualitative data that depends heavily on personal observation and judgement, but which is complemented by elements of quantitative data. However, it must be noted that, though outcomes are strongly indicated, they cannot be absolutely proven to be causally linked to the MSc scheme, nor can many of 
the reported benefits be truly measured. Nevertheless, there is sufficient triangulation across the various data sources to give a reasonable level of confidence about beneficial effects for the company, individual students and the university.

The study's population is the students who comprise the six cohorts that entered the HP MSc SQM programme between its inception in 2010 and 2015: in total 114 students, though feedback has only been obtained from the first five cohorts, because the most recent cohort, who only commenced their studies in the early summer of 2015 are not yet in a position to judge its efficacy. At the time of writing, approval has just been granted for the funding of a seventh cohort.

\section{Identifying the Need}

HP has a defined quality ecosystem (see Figure 1), which enables the company to:

a. "Recognise and accommodate business needs by means of an integrated approach to continuous improvement.

b. Exploit already existing employee skills such as Internal Assessors, Green Belts, Black Belts and Master Black Belts.

c. Continually develop our competencies by means of a tailored focused improvement curriculum" (Maguire, 2009).

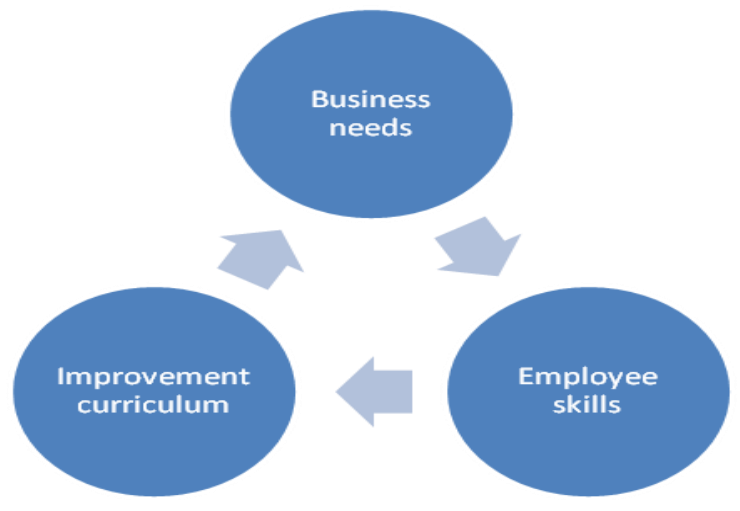

Figure 1: The HP Quality Ecosystem (Source: Maguire, 2009)

This ecosystem seeks to capitalise on the synergies between the needs of the business and the investigative and creative skills brought by quality improvement specialists, such as the various levels of Belts in the LSS hierarchy. As improvement opportunities are identified and actioned, the skills of these Belts are tested and mature. Cycles of improvement build confidence, but also allow knowledge and skills gaps to be identified. So, Belts' competencies are developed by formal training, mentoring and practice.

However, in 2009 senior TCE\&Q managers in HP identified a gap in the development ladder of their LSS Belts. They felt that the development of more effective academic research and critical thinking skills, coupled with a broader and deeper understanding of topics around strategic quality management, would provide a cadre of enhanced Black Belts, who were better suited to address the demands of strategic improvement projects. It was believed that this would improve the robustness of problem analysis, while also helping Belts to take a wider and more informed view to the design of possible solutions. This was felt to be particularly important for those undertaking projects with a scope that crossed the boundaries of separate business units, this being an increasingly frequent occurrence, given the complexity and size of the organisation. 
HP identified a set of desiderata which, they anticipated, would address these aims:

a. Enhancement of learning beyond HP's BB training programme through a considerable extended syllabus, leading to a Postgraduate degree.

b. Broadening of both knowledge and perspective though access to University knowledge resources, e.g. Professors, Libraries, etc., which would enable better leverage of knowledge for practical application within the company.

c. External accreditation of candidates' learning via partnership with a reputable University so that the qualification earned would be externally and internationally recognised.

d. Student projects published as dissertations and articles where applicable within the constraints of business sensitivities.

In the context of quality management professionals, such as LSS Belts, there is a need for technical analytical and investigative skills to be combined with creative problem-solving and change implementation. However, the balance between the technical and adaptive skills will vary according to the level of complexity of improvement problems being tackled.

According to Hilton and Sohal (2012, citing Hooper \& Devine, 2002) "the quality professional needs to re-invent their role in an organisation and move from 'quality control' to 'interpreters of business strategy' and drive the integration of all quality processes, metrics, tools and accountancy systems to optimise the performance of all departments". Thus, to operate effectively at a strategic level, quality activists, such as Black Belts and Master Black Belts, need a stronger awareness of both external good practice, identified via the academic literature base, and formal research methods training to ensure better awareness of the limitations of their investigations, more robust design of improvement projects, and hence to achieve better impact and longevity.

As LSS trained staff move up through the Belt development ladder, it is expected that their passion for improvement will be constantly regenerated, their communications and teamworking skills are enhanced; analytical skills which facilitate deployment of improvement within the complex political environment of the organisation are extended and their viewpoint widens to encompass the broader issues of societal influence and sustainability. In other words, they move from focus on numerical measurement to an understanding of the necessary soft skills to lead change in today's global organisation.

Within HP, at each step of the LSS training ladder, staff undergo formal training, either via online courses or face-to-face teaching. The training is then applied in the workplace as trainees undertake improvement projects, all the while mentored by those further up the Belt ladder. This is, of course, consistent with Kolb's Experiential Learning Cycle (1984). While many Belt-trained staff remain engaged in quite local improvement activities, a proportion move up to the next level, receive further training and then tackle projects with wider influence, thus forming a pyramid of quality professionals with increasing skills, who are able to mentor those below them and effect change at an increasingly strategic level.

However, as they progress to the more strategic levels, the types of skills and competencies required alters to ones that are less amenable to development via training and more demanding of the kind of intellectual "stretching" that comes with university study at postgraduate level. As Belts seek to tackle more complex situations, they have to understand and diagnose the effects of ever more fuzzy and intricate interactions, but ones that are also 
more difficult to measure reliably and accurately. Therefore, a greater awareness of the limitations of approaches to researching cause and effect becomes increasingly important, particularly where soft issues come to the fore. Similarly, courses of action leading to improvement tend to become less clear-cut. There are more options, more interactions, less certainty and hence greater potential for "emergent properties" or "knock-on effects" to arise.

The concept of an emergent property arises from studies of phenomena in the natural world (O'Connor, 1994), but is also well-known in various engineering domains, where it can be clearly seen in some physical systems. It rests on the fact that some features or behaviours of a system only become active once a set of condition or elements come together, sometimes only when the system becomes fully operational. Thus, these properties will be undetectable and unprovable by testing at component or sub-system level.

Emergent properties can be desirable and planned. Thus, for example, the intended function of a bicycle as a transportation device only emerges once the build is complete and a rider gets on. Until that point, while all the components and sub-systems may have been tested, there is no means to definitively prove the transportation function, even though it can be theoretically predicted.

However, it is frequently the case that emergent properties are undesirable and not foreseen. In fact, the more complex the system, the more likely this is to occur. In the case of business improvement projects, this will also be true. A Green Belt project will tend to be focused on a specific product or process and will thus be of limited scope and will have clearly defined boundaries and goals. In contrast, projects undertaken by Black or Master Black Belts may involve multiple processes, cross-functional and operational boundaries and have significant levels of socio-technical complexity, which may include a multiplicity of cultural variations across its sphere of influence. As a result:

a. Cause and effect linkages become more difficult to determine with confidence;

b. Pivotal intervention points are more difficult to identify;

c. Lines of ownership and control may be tangled;

d. Business interests and drivers may be in conflict across functional or business unit boundaries.

Thus, the core technical tools in the Black Belt toolbox are no longer capable of addressing the demands being placed on them. This is not to say that there is anything wrong with these tools, but rather that they need to be supplemented, just as the Green Belt toolbox is expanded by Black Belt training. It is the role of the MSc SQM programme to help Black Belts develop additional skills and techniques so that they are better fitted to cope with the demands of projects with organisation-wide scope, high complexity and considerable business impact.

\section{The Degree Programme: development and experience}

The University of Portsmouth Business School has a well-established MSc in Strategic Quality Management (SQM). It operates as a traditional taught course and is also offered in Distance Learning mode, with academic input being provided via the University's Virtual Learning Environment (VLE) and online classrooms. The syllabus of this degree was a good match for the developmental needs that had been identified within HP's LSS programme. Of particular interest was the strategic focus of the degree, which seeks to bridge the gap between 
knowledge of quality management tools and techniques and their strategic application within an organisation. This presented a good opportunity for HP to add an additional step to the development ladder for their Quality Improvement Professionals (see Figure 2).

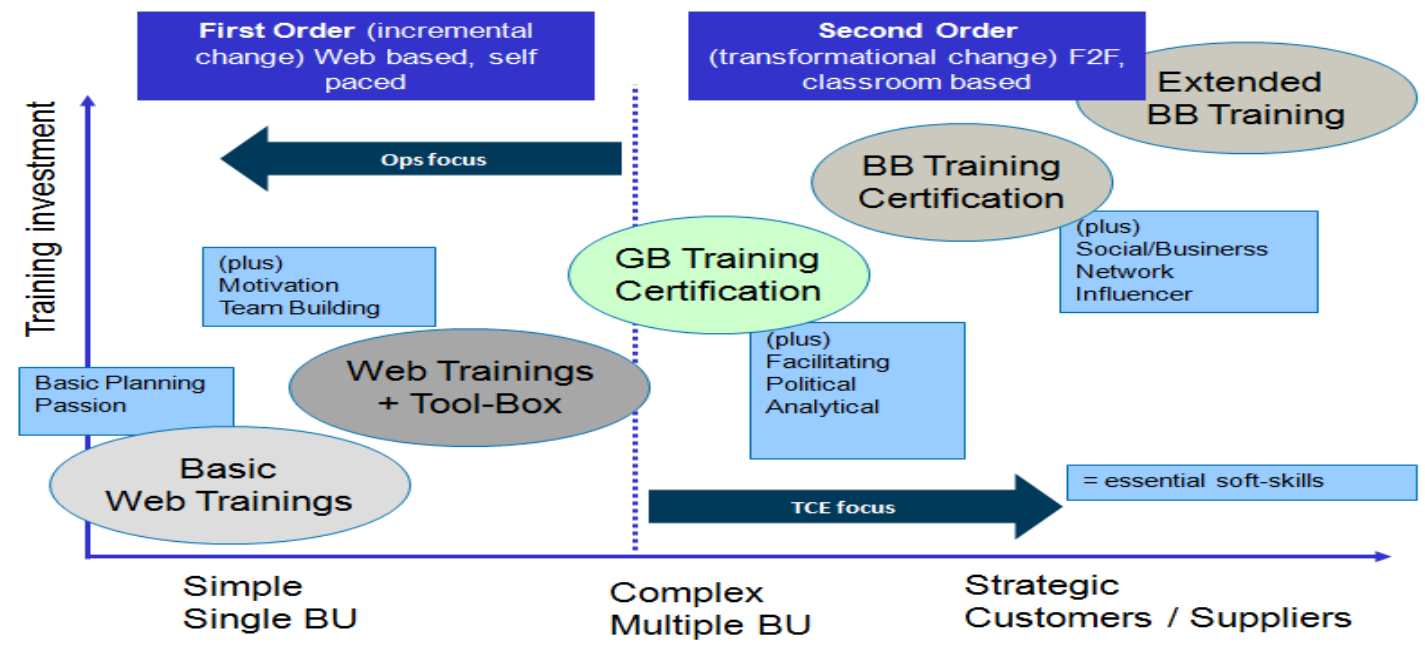

Figure 2: Staff Capability Development vs Impact Achieved (Source: Maguire, 2009)

An analysis of the syllabi for the Black Belt training against the MSc SQM identified significant overlap in some areas. As a result, it was decided that it would be possible to grant accreditation for this prior learning (APEL) with only a fairly small amount of "bridging". Thus the HP candidates are able to claim exemption from 60 credits (which is one third of the total of the 180 credits required for the MSc) via a combination of several short assignments relating to the bridging material and evidence of successful completion of $\mathrm{BB}$ training (candidates are not required to have achieved BB Certification). Once this is achieved, they undertake the full diet of study and assessment faced by all students on the established Fulltime and Distance Learning routes through the degree.

The total duration of the long-established Distance Learning version of the MSc is 26 months, though some vacation periods are built into the study schedule. However, the HP design team set the target for completion at 18 months, which was deemed to be possible due to the accreditation relating to the previous BB training. In practice, the experience of the first cohort very strongly indicated that this ambitious timetable was placing unnecessary strain on the candidates, distracting them somewhat from their work responsibilities and reducing the developmental benefits of the course. In particular, the individual dissertation (these were closely linked to a company improvement project relevant to a given student) was being undertaken through the summer period, which caused difficulties as data gathering activities clashed with the most popular time for company staff to take holidays. It was therefore decided to extend the duration to 22 months for subsequent intakes. The experience of succeeding HP cohorts indicates that this has successfully mitigated the timing and workload problems encountered by the first group. Despite this lengthening of the schedule, the HP students complete in four months less than those on the normal Distance Learning route. No formal vacation periods are planned into the HP schedule, so it is up to individual candidates to manage their work, study and family responsibilities in such a way as to permit them to achieve a reasonable work-life balance. HP students remain subject to the normal University regulations and receive no special treatment or exemptions to those regulations, which provide to all students a means to address the effects of extenuating circumstances, whether arising from personal circumstances or work pressure. 
The course is delivered via a combination of face-to-face Master Classes and distance learning, though this was not the original plan, which was to deliver the Master Classes via a virtual classroom. This approach would have been consistent with the operation of the majority of team-working activities within HP. Unsurprisingly, given the size and geographical spread of the company, teams tend to be diverse, often being composed of members from a distribution of cultures, geographies and time zones. Nevertheless, to prove the concept, it was decided that Master Classes for the first cohort should be a live classroom experience, with delegates travelling to Portsmouth for a week in May and another in September. Because of strong positive feedback both from students and teaching staff, the two weeks of face-to-face Master Classes have been retained as a core element of the scheme.

\section{$5 \quad$ Student Profile}

Participation in the programme is voluntary. Several months prior to the commencement of a new group of students to the degree programme, the TCE\&Q Lead circulates details of the course to Black Belts. All interested candidates have to make a case for their inclusion, which includes the likely contribution that their individual research project would be expected to make to the company, to gain support from their manager and to pass an internal HP selection process to gain HP's sponsorship. Only then can they submit an application to the University. However, they are also subject to the University's normal academic admissions criteria, so that HP sponsorship does not guarantee a place on the course. Close working relationships between key members of the HP selection panel and the University's Course Leader have ensured that rejection at the formal application stage is extremely rare.

Cohort by cohort, the profiles of the groups has altered in its nationality mix. The first HP MSc cohort comprised 21 candidates who were drawn exclusively from across HP's Europe, Middle East and Africa (EMEA) operational domain, originating from the UK, Germany, Holland, Romania, Hungary, Spain and Israel. In subsequent cohorts, the catchment has been broadened to encompass the whole of the world-wide HP organisation. Across the first six intakes, 27 nationalities were represented by candidates working in 26 different countries, spread across the three HP regions (EMEA, Americas (AMS) and Asia Pacific and Japan (APJ)).

Table 1: Students by HP Region

$\begin{array}{llllccll}\text { Region } & \text { Total } & \text { Cohort 1 } & \text { Cohort 2 } & \text { Cohort 3 } & \text { Cohort 4 } & \text { Cohort 5 } & \text { Cohort 6 } \\ \text { AMS } & 23 \% & 0 \% & 13 \% & 5 \% & 33 \% & 41 \% & 48 \% \\ \text { APJ } & 21 \% & 0 \% & 19 \% & 29 \% & 44 \% & 29 \% & 10 \% \\ \text { EMEA } & 56 \% & 100 \% & 69 \% & 67 \% & 22 \% & 29 \% & 43 \%\end{array}$




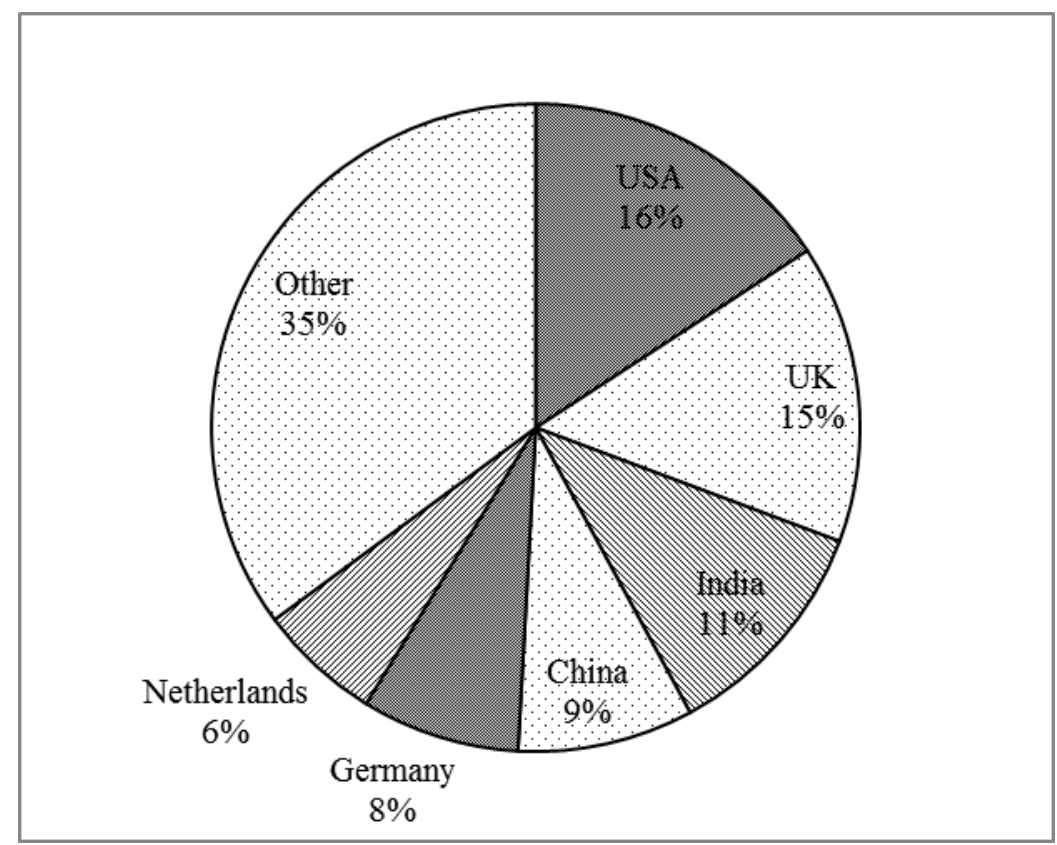

Figure 3: Student's Work Base ('Other' includes 20 countries each contributing less than $5 \%$ of the student population)

Candidates range in experience from those who were undertaking the Black Belt training in parallel with the MSc, to Certified Black Belts and up to Master Black Belts. All three of the Master Black Belts who had been involved in the original development of the scheme joined the first cohort, having subjected themselves to the same selection process undergone by other candidates. Likewise, there is a considerable range of ages. Though most are in their 30s or 40s, each group has included one or two "elder statesmen". While questions might be raised about the cost effectiveness of including staff who are within sight of retirement, these older entrants have brought with them a wealth of experience, both of quality matters and life; they brought huge levels of enthusiasm for the opportunity to learn, but typically coupled with a very engaging degree of humility about their own capabilities. Thus they have provided not only thoughtful input to class debate about quality matters, but have also often been the ones who had the confidence to express doubts or challenge academic assumptions and so stimulate discussion about why the teaching team believed something was important to their development and, even if not immediately obvious, how it would ultimately be of value to the company.

\section{Delivery Experience}

The expectation was that learning during the Master Classes would be based on a fairly typical, if rather more concentrated, university lecture delivery, though with breakout sessions to allow students to debate how topics might be applied to add value within HP by comparing and contrasting different ideas from academic theory and research with personal experience in practice. The Master Class weeks are highly intensive, so as to maximise the benefit against time out of the office and the associated costs.

However, in practice, there were unforeseen additional benefits from having the students physically co-located. Having little knowledge of the internal workings of the company, the academic team had not considered the effects of the way in which HP has grown over the years. HP's company culture is encapsulated in the "HP Way", an approach 
which has sustained the organisation over many years. Such an organisational culture arises from a set of shared assumptions that a group has learned to adopt to meet both internal and external challenges. Once established and proven to work well, it can be sustained in the longterm and taught to new members (Gupta, 2011, p. 513). Since HP's growth has involved a significant number of corporate acquisitions, the integration of acquired companies into the "HP Way" is an ongoing process.

There is sometimes a clash of cultures between academia and industry. This was explored by Garavan (1997). He reported that training tends to be completed in a shorter timescale than education and that it has more focussed outcomes, and that learning has broad goals that recognise many ways of thinking and doing. Thus, the development of employees is not simply a matter of delivering tightly-defined knowledge or skills, but rather in expanding an individual's potential in dimensions that may only be quite generally defined, and where unforeseen outcomes may occur. So, while there may be a set of desired developmental outcomes which it is anticipated will be seen in all candidates, there will also be a spectrum of other skills, intellectual or attitudinal changes that will vary from individual to individual, both in depth and combination.

More recently, Clinebell and Clinebell (2008) reviewed the tensions that arise when seeking to maintain academic rigour, while also delivering real-world relevance. They cite DeAngelo, DeAngelo and Zimmerman (2005) who reported that business students were being taught "how to structure their thinking" and "educating students by providing a conceptual framework that can be used to analyse a wide variety of future business problems". Clearly, this view is entirely in keeping with the desiderata set out by HP at the start of the collaboration, but it is also inherent in the original design intent and delivery mind-set of the MSc SQM's academic team. As a result, adaptations necessary to the content and delivery were quite minor and tended to relate more to practicalities than subject matter, with the main change relating to ensuring that there was only minimal overlap in delivery where topics had already been covered in Black Belt training.

Interestingly, this tension does become visible in the classroom, when some students in each cohort challenge the lecturers about why they were "wasting" time on irrelevant subjects, particularly in respect of the strong focus on research methods. While by the end of the course, students have clearly come to understand the value of such topics, in the early days, some evidently struggle to switch their expectations from a training context that delivers tools and techniques that are fairly immediately useable on the job, to the longer-term, more applied developmental learning that comes with Master's level study.

It has also become apparent that students gradually developed an appreciation that knowing something in practice was not the same as what we might term "academic knowing", where there is no "one version of the truth". Instead, theory becomes a jumping-off point for exploring how situation and context might drive adaptation of approaches to achieve success. This is where the drive to explore and critically evaluate academic literature comes to the fore, especially where findings show variation or even conflict.

\section{$7 \quad$ Building a Community}

Some of these students had come into HP when their companies were acquired and the cultural legacy from these acquired companies has persisted, sometimes over many years, even though it has been overlaid by HP corporate culture. Examples that were visible in the 
MSc cohorts were Compaq and, more recently, EDS. Bolboli and Reiche (2014, p. 331) highlight that an organisation's cultural patterns are influenced by its members throughout their interaction and this can consequently form a new cultural environment. Thus it is necessary for the company to constantly seek to reinforce the core elements of its desired culture, in this case, the HP Way.

During breakout sessions, in-class discussions, coffee breaks and meal times, it quickly became apparent that an additional layer of learning was occurring as these delegates examined their differing experiences and viewpoints outside the constraints of formal meetings and started to explore opportunities to share practices, with a view to achieving some form of consensus on best, or at least, better, practice. A student encapsulated this view saying "benefit comes from the face-to-face lectures and the vast experience level that the other students of the class bring to the discussions that have occurred in Portsmouth. We have developed new working relationships and I have a much better grasp of the global reach and challenges that we must conquer in HP". Observations of both the Full-time and standard Distance Learning routes of the MSc SQM, indicate that the value-added from such sharing is considerably less when students have to censor their input to protect company confidentiality; a factor that does not occur in the all-HP cohorts.

This experience can be compared with that observed in a large MSc programme in the engineering domain, run under the auspices of the UK Integrated Graduate Development scheme (IGDS), (one of the authors of this paper was involved in the development and delivery of an IGDS programme for some 15 years, prior to her current appointment). The IGDS MSc programmes focus on graduate engineers in a specific sector (for example, aerospace or automotive). They are structured somewhat similarly to the HP scheme, but have considerably more contact weeks. Material is packaged in up to 12 subject modules, each of which is associated with a week-long teaching block. Once the taught modules have been completed, students undertake an individual research dissertation. However, there are other distinct differences. Students have some choice in which subject modules they take and in the order they take them, so students tend not to progress through the course in a defined cohort. Furthermore, there is no guarantee that a given run of a module will be taken only by students from a single company, though this does happen sometimes at the request of the sponsoring company, but usually only where there is sufficient volume to make it economic to run what are, in effect, both a public and private delivery. Where "private" deliveries occurred, the impact of students being able to engage in frank and open discussion was also noticeable (former IGDS Administrator, University of Warwick, personal communication, April 2015).

The in-class sharing during HP MSc SQM classes has been further extended by the formation of small (3-6 persons) study groups designed to help the students support their own learning during the Distance Learning phase of their studies. The cross-cultural teams helped to bring the good perspectives of team work and learning based on the experiences that people have in different countries and geographic locations (Xiaojing et. al., 2010).

HP operates a 'LSS Community forum', that is a community of LSS trained employees who meet (virtually) to share learning and good practice, but, within this, the MSc students initially started to gel into a quite cohesive specialist Community of Practice, drawn together by the common experience and challenges of the MSc. The successive cohorts are forming longitudinal links, with members of the first cohort acting as "buddies" to the second and so on. A student stated "It's very helpful to have a study group of peers. It's been helpful to have occasional interaction with previous graduates of the programme at HP". It is anticipated 
that this group will become a recognised expert resource, able to offer a sort of consultancy provision to the wider Black Belt cadre.

Wenger (1998) states the presence of Communities of Practice to be a facilitating factor for organisational learning. He suggests that Communities of Practice form naturally within organisations, to facilitate a more social approach to learning. These communities may be based around less formal networks of individuals sharing knowledge of particular work topics or expertise. Collison and Parcell (2004) further built upon this by stating that Communities of Practice are the "stewards of knowledge and competence". However, Wenger and Snyder (2000) describe the essential nature of Communities of Practice to be informal and selforganising, including the voluntary membership of individuals, so perhaps there will be those who challenge whether this MSc student cluster is actually a Community of Practice by the strict definition. In addition, Amin and Roberts (2008) have taken this further to contend that Communities of Practice, as originally defined, are simply one form of "knowing in action". Nevertheless, however it is labelled, this group does seem to be exhibiting the beneficial characteristics reported for such communities and so steps need to be taken to foster its survival in the longer term.

\section{Characteristics and Achievement}

The first cohort of HP Black Belts graduated in the summer of 2012, and they have been followed by successive cohorts annually. The marks of individual students have tended to show a clear upward trajectory in their academic development through the course and overall they achieved some very creditable final results. Although there have been a few drop-outs, these have been either for personal reasons, or, more recently, as a result of the major workload demands relating to preparations for the HP company split that occurred in November 2015.

Indeed, when considering the three years (2012-14) of graduating students from all routes through MSc SQM, the results of the first three groups of HP students compare favourably with those on the other delivery routes, in that an unusually high proportion have earned one of the higher classifications. MSc classifications are broadly aligned with British undergraduate degree classifications, so that a Distinction, like a First Class Honours, is awarded for marks of 70\%+, while a Merit equates to an Upper Second Class (60-69\%) and a Pass covers both Lower Second and Third Classes (40-59\%).

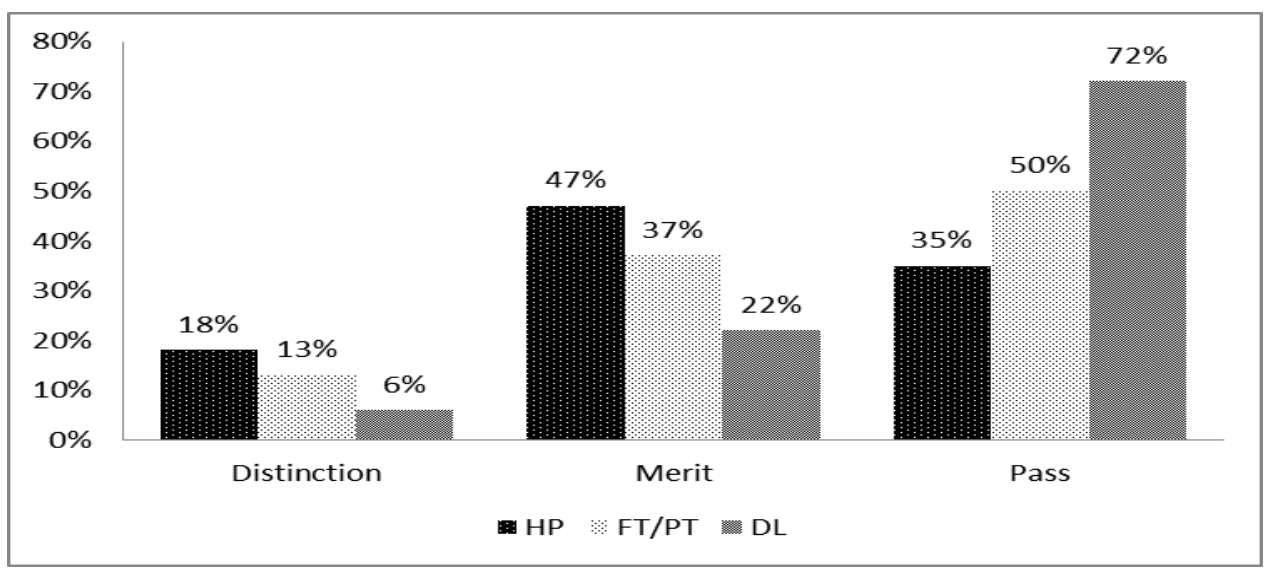

Figure 4: Comparison of Degree Classifications Earned by Study Route (2012-14 Graduates) 
It is worth noting that educational backgrounds of the HP students were quite varied. While the majority entered with undergraduate degrees (in a variety of subjects), there were many who had already gained a Master's degree (or even two!) in another subject.

However, in some respects, the most remarkable were the few who had not had the opportunity for university study after leaving school. Their admission to the course was justified by significant management level experience. While they were clearly excited about the prospect of studying for an MSc, there was a very strong overtone of fear, partly of the unknown and partly that they would not be able to cope. Nevertheless, most succeeded, and succeeded well; indeed, one of these non-graduate entrants earned a Distinction overall in the MSc. One said "I left school at 15 and so was slightly nervous at the thought of studying again, but the course made me realise how much experience I have and has allowed me to put into place the theory behind it. I have been taught so much that I can bring to my job".

\section{How is the HP MSc SQM Delivering Value?}

While a variety of intangible benefits have been highlighted above, this is not sufficient to justify continued investment in the scheme; there must be real measurable business gains. Feedback from HP indicates that the course has contributed to significant additional process improvement Return on Investment (ROI) derived from a set of strategically aligned initiatives managed by the MSc students.

The MSc SQM programme helps to increase Employee Engagement through employee development. There are intangible benefits by the added knowledge they acquire throughout the course in addition to the reflection of the work experience. The course also adds to and enhances the application of LSS tools learnt during the Black Belt training and how to apply them. It enhances the experience by helping the candidates apply research methodology skills to ensure proper assessment of risks and benefits of alternative approaches and hence delivers a research project that adds value to HP. This was confirmed by a recent in-house study (Verdurmen, 2015), when current and past students on the programme were questioned regarding the impact of the programme. Respondents suggested that they had gained: "the ability to see the bigger picture and think more strategically"; "verification of facts demonstrating a more critical thinking approach" and "active application of Research Methods". Verdurmen's research also revealed that graduates identified the one-to-one relationship with their dissertation supervisor as being highly significant in helping them truly internalise the knowledge gained, thus making it ready for deployment in their work.

The perceived value of the course from the company's viewpoint falls into three categories: knowledge, tools and employee development. Together these combine to increase employee engagement and hence, boost these individuals' effectiveness so as to deliver their goals and their organisation's goals to satisfy the internal and external customers.

The basic toolset that candidates have already assembled through their Belt training is augmented through the topics covered in the course, but more importantly, exposure to a wide range of academic research studies, via the literature, allows them to observe, albeit secondhand, the effects of tools and techniques in use in a much wider variety of situations and contexts than any single person would be able to do during the course of a normal professional career. This enables them to better comprehend the potential for adaptability offered by many common quality improvement approaches. Furthermore, use of external 
research points out both critical success and failure factors (CSFs and CFFs) and links these to a context in a way that makes it possible to understand likely cause and effect relationships. This, in turn, adds another set of tools to the student's collection: diagnostic tools to be used in the soft systems environment where culture and situation hold sway over more clear-cut operational factors. This is exemplified by one student's statement "I have learned a new way to learn. I have learned of the extensive wealth of information and studies that exist that I can read to try new ideas and leverage what others have learned. In the past, I would use my 25 years of experience to base my next steps on, plus sometimes reaching out to a respected colleague. Now I find myself seeking articles or books as well”.

The fact that there is rarely complete agreement in the academic literature may initially seem threatening, especially to those who come from a strong quantitative background, which is typical of those who have progressed through the Belt system and are thus used to the idea that numbers can be analysed to give definitive answers. However, in the realm of the complex, cross-functional, cross-business unit, multinational strategic projects that these candidates are being prepared for, things are unlikely to be either simple or prone to straightforward qualitative analysis. Therefore, the ability to use the literature to gain a perception of how reactions to applications of various approaches may vary according to context and culture allows the student to work more effectively through an understanding of potential pitfalls or sensitivities. The literature does not give all the answers, but raises awareness that helps the reader to more safely plot their way through an unfathomable landscape to a more reliable conclusion. In addition, the development of stronger methodological research skills makes each activity more robust, thus enhancing the chances of sustainable success and reducing wastage and overhead costs.

From the academic viewpoint, it is believed that the "enforced" paradigm shift from business thinking to academic review and reflection, coupled with the implied permission to explore the literature without the pressure of looking for direct business returns, has changed the attitudes of these Black Belts. One student commented 'Students were 'forced' to read material that they would not have previously read, which has given some additional insight into other good practices" and another stated "the SQM course has been an eye opener, it opens the door to a new world outside HP”.

Their approach to their improvement projects has shifted somewhat, so that they are focussed not merely on the ultimate financial gain, but more on the holistic set-up of the change project, with a view to better assuring long-term sustainability of solutions, rather than just quick wins. This is not to say that they are ignoring the financial outcomes, but instead that they are taking a longer view of how the ROI should be judged and giving greater consideration to other factors critical to success. This is, of course, entirely in keeping with Deming's view. They expend more effort on ensuring that the problem is properly understood, they use the literature to assess what others have done, and with what effect, and they evaluate the possible methods to be used to select the most appropriate. Thus the front end of their improvement project gets more attention than before, which has knock-on benefits in the smooth running and impact of the later stages, whilst not delaying the project overall. Hence, this results in completed projects that deliver stronger and more robust outcomes for the organisation, so that the overall ROI, taking into account both tangible and intangible benefits, is high.

In addition, there is evidence of knowledge exchange from the MSc students to their colleagues. One student reported "In one of our other teams I actually passed on some of my 
experience and drove people to look beyond the HP-world that they probably know too well by now, and they explored, not necessarily academic, but nonetheless, valuable market and area-development information, leading them to identify some innovative and game-changing ideas to pursue".

It is clear that these HP MSc graduates now not only have a stronger technical background in quality, but that they have also gained a substantial enthusiasm boost. Indeed, informal conversations with students in the more recent HP intakes confirm that many wanted to enrol on the course because of what they have heard from members of the earlier cohorts. It will be interesting to see if this enthusiasm might have an effect on more junior members of the LSS programme, perhaps through graduates mentoring novice Green Belts through Certification, or in encouraging more experienced Green Belts to wish to develop their skills to Black Belt level.

These points are echoed by student comments in the feedback questionnaires. Indeed, one of the recent graduates stated that the effect of taking the course had been "better than a pay rise!", while another reported that it was a "Challenge to beliefs and behaviours, takes us outside our complacency". A third said "The content built up and into my Black Belt course, but the perspective I got on the content is quite useful. I enjoyed the case studies \& ways to put theory into practice as well as opportunity for best practice sharing".

Another outcome has been increased employee engagement, most clearly evidenced by the fact that a high proportion of the students have progressed into new roles within the company that capitalise on their new knowledge and skills. One student reported "it was partially thanks to my participation in the SQM course that I was chosen to take on the role of ... Quality Strategy and Planning lead within the ... Quality organization" (detail omitted to protect anonymity). Another said "the course helped me with my career development within HP as well as being an extremely challenging, therefore rewarding experience”. In one study group, by the halfway point in their studies, three members had moved into more specialist roles. In another cohort, within a year of graduation, three graduates had taken on world-wide responsibilities. This is clear evidence that the enhanced capabilities of those who have engaged in the MSc SQM programme are being capitalised upon by the business.

An additional, and somewhat unexpected, outcome is that two graduates from the first cohort are now undertaking doctoral studies (one is registered for a $\mathrm{PhD}$ and the other a DBA). Both felt challenged by their MSc experience and have chosen to progress their academic development part-time and self-funded. Several other graduates are actively considering possibilities for further study.

\section{Conclusions}

This collaborative scheme has delivered significant benefits to HP and seems set to expand and grow in the future. In addition, there is an element of feedback into the wider MSc Strategic Quality Management as teaching staff complement their academic knowledge with understanding of the realities of LSS implementation in a real-world environment. The cultural and experiential diversity of the HP students is generating a significant level of broadening of the views of all concerned. Perhaps it is appropriate that the final words should come from some of the scheme's graduates. One said "I've found the course to be very interesting and relevant to my role in HP. It has been enjoyable to step back from the day-today work and look at higher level questions of how and why". Meanwhile, another 
claimed "SQM has given me whole new perspectives, has equipped me with new methods, and connected me with some extraordinary colleagues, and step by step we're leaving our prints in the work we perform".

This model has obvious potential for adoption by other companies, providing they are large enough to release sufficient staff to form a viable group (a minimum of 12 candidates, though 15-20 will tend to generate richer debate and mutual support). While the current scheme is designed for Black Belts, who are able to gain advanced standing, it would be quite simple to adjust the content and delivery to suit the needs of candidates who needed to study the full MSc SQM syllabus, for example, Green Belts, auditors or quality managers.

Another model that has recently been launched is a staged one, where a company group initially commences by studying only for a Post Graduate Certificate (in effect, the first third of the MSc), via a combination of in-house face-to-face teaching and distance learning. On completion of this stage, company and students can decide whether to progress to the second phase (Post Graduate Diploma) and hence, by completing an individual dissertation, on to the award of the full MSc SQM.

The MSc SQM, with its suite of delivery modes, presents a range of opportunities for both organisations and individual students. This paper has evaluated how one custom variant is meeting the needs of a major international company in helping to develop and enhance the potential of their quality professionals.

\section{References}

Amin, A. \& Roberts, J. (2008). Knowing in Action: Beyond Communities of Practice, Research Policy, Vol 37, pp 353-369.

Bolboli, S.A. \& Reiche, M. (2014). Culture-based design and implementation of business excellence, The TQM Journal, Vol 26 Iss 4, pp. 329 - 347.

Bryman, A. \& Bell, E (2011) Business Research Methods, ( $3^{\text {rd }}$ ed.), Oxford University Press, Oxford, UK

Clinebell, S.K. \& Clinebell, J.M. (2008). The Tension in Business Education Between Academic Rigor and Real-World Relevance: The Role of Executive Professors, Academy of Management Learning \& Education, Vol 7, No 1, pp 99-107.

Collison, C. \& Parcell, G. (2004). Learning to Fly: Practice Knowledge Management from Leading and Learning Organizations, Chichester, U.K: Capstone.

Garavan, T.N. (1997). Training, development, education and learning: different or the same?, Journal of European Industrial Training, Vol 21 Iss. 2, pp 39-50.

Gupta, B. (2011). A Comparative Study of Organizational Strategy and Culture Across Industry, Benchmarking: An International Journal, Vol 18 Iss. 4, pp. 510 - 528.

Hilton, R.J. \& Sohal, A. (2012). A conceptual model for the successful deployment of Lean Six Sigma, International Journal of Quality \& Reliability Management, Vol 29 Iss. 1, pp 54-70.

Kolb, D.A. (1984). Experiential Learning: Experience as a Source of Learning, Prentice-Hall, New Jersey.

Maguire, P. (2009). TCE\&Q Strategy, Unpublished Hewlett Packard internal report.

O'Connor, T. (1994). Emergent Properties, American Philosophical Quarterly, Vol 31 No 2, April, pp 91-104.

Saunders, M, Lewis, P. \& Thornhill, A. (2012) Research Methods for Business Students, $\left(6^{\text {th }}\right.$ ed.), Pearson, Harlow, UK 
Verdurmen, A. (2015). Way(s) to improve articulation of tacit knowledge within the HewlettPackard organization, (Unpublished MSc dissertation) University of Portsmouth, Portsmouth.

Wenger, E. (1998). Communities of Practice, Cambridge University Press: Cambridge.

Wenger, E.C. \& Snyder, W.M. (2000). Communities of Practice - The Organizational

Frontier, Harvard Business Review January - February, Reprinted in Harvard Business

Review on Organizational Learning, Boston: Harvard Business Press.

Xiaojing, L., Shijuan, L., Seung-hee, L. \& Richard, J. M. (2010). Cultural Differences in

Online Learning: International Student Perceptions. Educational Technology \& Society, Vol 13 Iss. 3, pp177-188. 